\title{
Solar Flux Emergence Simulations
}

\author{
R.F. Stein · A. Lagerfjärd · Å. Nordlund • \\ D. Georgobiani
}

Received: 2 November 2009 / Accepted: 7 January 2010 / Published online: 19 February 2010

(C) The Author(s) 2010. This article is published with open access at Springerlink.com

\begin{abstract}
We simulate the rise through the upper convection zone and emergence through the solar surface of initially uniform, untwisted, horizontal magnetic flux, with the same entropy as the nonmagnetic plasma, that is advected into a domain $48 \mathrm{Mm}$ wide by $20 \mathrm{Mm}$ deep. The magnetic field is advected upward by the diverging upflows and pulled down in the downdrafts, which produces a hierarchy of loop-like structures of increasingly smaller scale as the surface is approached. There are significant differences between the behavior of fields of $10 \mathrm{kG}$ and 20 or $40 \mathrm{kG}$ strength at $20 \mathrm{Mm}$ depth. The $10 \mathrm{kG}$ fields have little effect on the convective flows and show small magnetic-buoyancy effects, reaching the surface in the typical fluid rise time from $20 \mathrm{Mm}$ depth of 32 hours. 20 and $40 \mathrm{kG}$ fields significantly modify the convective flows, leading to long, thin cells of ascending fluid aligned with the magnetic field and their magnetic buoyancy makes them rise to the surface faster than the fluid rise time. The $20 \mathrm{kG}$ field produces a large-scale magnetic loop that as it emerges through the surface leads to the formation of a bipolar, pore-like structure.
\end{abstract}

Keywords Sun · Dynamics · Magnetic fields · Magnetic flux emergence $\cdot$ Convection

Helioseismology

Guest Editors: G. Houdek, H. Shibahashi, and J. Zhao.

R.F. Stein $(\varangle) \cdot$ D. Georgobiani

Michigan State University, East Lansing, MI, USA

e-mail: stein@pa.msu.edu

D. Georgobiani

e-mail: dali@pa.msu.edu

A. Lagerfjärd · Å. Nordlund

Astronomical Observatory/Niels Bohr Institute, Juliane Maries Vej 30, 2100 Copenhagen, Denmark

A. Lagerfjärd

e-mail: anders।@astro.ku.dk

Å. Nordlund

e-mail: aake@astro.ku.dk 


\section{Introduction}

We have initiated simulations of the rise of magnetic flux through the near-surface layers of the solar convection zone. Numerous studies exist of the rise of a thin magnetic flux tube through the bulk of the convection zone (e.g., Moreno-Insertis, 1986; Choudhuri and Gilman, 1987; Fan, Fisher, and McClymont, 1994; Moreno-Insertis, Caligari, and Schüssler, 1994; Caligari, Moreno-Insertis, and Schüssler, 1995). These break down near the bottom of our simulation because the magnetic pressure exceeds the gas pressure and the tubes explode. Others have studied the rise of coherent, twisted, nonthin flux tubes through model convection zones (e.g., Dorch, 2003; Abbett et al., 2004; Fan, 2009). The rise of a coherent, twisted flux tube through the shallow layers of realistic solar convection zones has been studied by Cheung et al. (2008), Cheung, Schüssler, and Moreno-Insertis (2007), Cheung, Moreno-Insertis, and Schüssler (2006), Martínez-Sykora, Hansteen, and Carlsson (2008, 2009). We are undertaking a complementary study: the rise and evolution of untwisted, horizontal flux with the same entropy as the nonmagnetized ascending plasma, carried by upflows from a depth of $20 \mathrm{Mm}$. Here we present some preliminary results from these simulations.

\section{Simulation}

Our domain is $48 \mathrm{Mm}$ wide and from the temperature minimum to a depth of $20 \mathrm{Mm}$ (Figure 1). We solve the equations for mass, momentum, and internal energy in conservative form plus the induction equation for the magnetic field, for fully compressible flow, in three dimensions, on a staggered mesh. The code uses finite differences, with sixth-order derivative operators and fifth-order interpolation operators. The grid is uniform in horizontal directions with resolution $95.24 \mathrm{~km}$ and non-uniform in the vertical (stratified) direction with resolution of $12 \mathrm{~km}$ near the surface increasing to $75 \mathrm{~km}$ at the bottom of the domain. Transformation between uniform index space, where the derivatives are evaluated, and the nonuniform physical grid is achieved by multiplying by the Jacobian of the transformation. The staggered mesh increases the order of the derivative operators for the same size stencil, but the physical variables are not aligned in space and need to be interpolated using fifth-order interpolation operators. Time integration is by a third-order, low-memory, Runge-Kutta scheme (Kennedy, Carpenter, and Lewis, 1999). Parallelization is achieved with MPI, com-
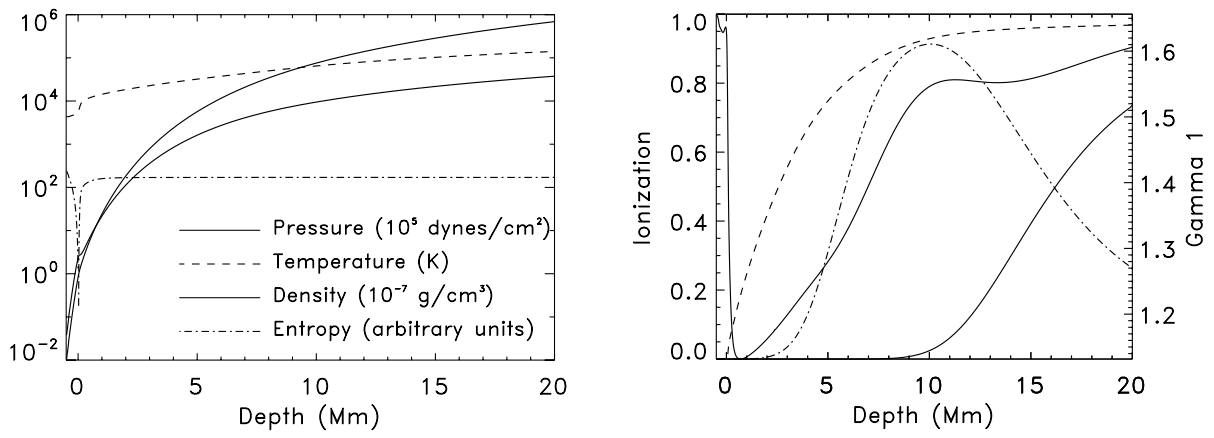

Figure 1 Mean atmosphere of the model. The domain includes all of the Hydrogen and Helium I ionization zones and most of the Helium II ionization zone (H dashed, He I dash-dot, He II dash-dot-dot-dot, $\Gamma_{1}$ solid). It covers only $10 \%$ of the geometric depth of the solar convection zone, but half of the pressure scale heights. 
municating the three overlap zones that are needed in the sixth- and fifth-order derivative and interpolation stencils. Because of the staggered mesh, $\operatorname{div}(\mathbf{B})$ is conserved to machine precision. However, numerical errors in $\operatorname{div}(\mathbf{B})$ that slowly accumulate stochastically must be cleaned at intervals. Ghost zones are loaded at the top and bottom boundaries to permit the use of the same spatial-derivative scheme at the boundaries as in the interior. Horizontal boundary conditions are periodic, while top and bottom boundary conditions transmit the convective flows but partially reflect waves. Inflows at the bottom boundary have uniform pressure, specified entropy and damped horizontal velocities. Outflow boundary values are obtained by extrapolation. The magnetic field is made to tend toward a potential field at the top. That is, the ghost zones are loaded with a potential field calculated from the values of the vertical field just inside the boundary. At the bottom the magnetic field is given a specified value in inflows and extrapolated in outflows. The electric field in the ghost zones is calculated from the magnetic field and the velocities (which are taken to be constant at their values on the boundary at the top and have zero vertical derivative at the bottom) in the ghost zones. The magnetic field is updated from its temporal derivative, which is the curl of the electric field. The code is stabilized by diffusion in the momentum, energy, and induction equations.

Ionization energy accounts for $2 / 3$ of the energy transported near the solar surface and must be included to obtain the observed solar velocities and temperature fluctuations (Stein and Nordlund, 1998). We use a tabular equation of state, which includes local thermodynamic equilibrium (LTE) ionization of the abundant elements as well as hydrogen molecule formation, to obtain the pressure and temperature as a function of log density and internal energy per unit mass.

Radiation from the solar surface produces the low-entropy, high-density fluid whose buoyancy work drives the convective motions, and it also provides us with our only information on what is occurring there. Since the optical depth is of order unity in these regions, neither the diffusion nor optically-thin approximations are valid. We calculate the radiative heating/cooling by solving the radiation transfer equation in both continua and lines using the Feautrier method (Feautrier, 1964), assuming Local Thermodynamic Equilibrium (LTE). The number of wavelengths for which the transfer equation is solved is drastically reduced by using a multi-group method whereby the opacity at each wavelength is placed into one of four bins according to its magnitude, and the source function is binned the same way (Nordlund, 1982; Stein and Nordlund, 2003; Vögler, Bruls, and Schüssler, 2004). The conversion of energy transport from convection to radiation occurs in a very thin thermal boundary layer, which must be resolved (grid size less than $15 \mathrm{~km}$ ) in order to obtain the correct entropy jump and the solar convective adiabatic. This radiation solution is validated by comparing a few snapshots with a detailed solution having a large number of wavelength points and many more angular quadrature points. Calculated spectral-line widths, Doppler shifts, and asymmetries from the simulations are in excellent agreement with observations (Asplund et al., 2000).

At supergranule and larger scales, the Coriolis force of the solar rotation begins to influence the plasma motions, so $f$-plane rotation is included in the simulation. Angular momentum conservation produces a surface shear layer with the surface (top of the domain) rotating slower than the bottom of the domain, as observed in the Sun.

We do not know the properties of the magnetic field at the depth of $20 \mathrm{Mm}$. We have investigated the behavior of untwisted, horizontal flux with the same entropy as the nonmagnetized ascending plasma, advected into the computational domain by upflows from a depth of $20 \mathrm{Mm}$. Wherever there was inflow at the bottom, uniform horizontal field in the $x$-direction is advected into the computational domain. We have calculated three cases, with 
field strength at the bottom of 10,20 , and $40 \mathrm{kG}$. In the outflows the field values are extrapolated. At the boundaries between in- and outflows, the field magnitude is smoothly varied. The boundary conditions are applied to the electric field from which the temporal derivative of the magnetic field is calculated. The initial state in all three cases was the same snapshot of field-free hydrodynamic convection.

\section{Flux Emergence}

Upflows carry the field toward the surface, and for the case of the stronger fields, magnetic buoyancy increases the speed of ascent. The typical time for fluid to rise from a depth of $20 \mathrm{Mm}$ to the surface is 32 hours. The field in the stronger $(20 \mathrm{kG})$ case reaches the surface in about 20 hours, assisted by magnetic buoyancy. The field in the weaker $(10 \mathrm{kG})$ case reaches the surface in the fluid travel time of 32 hours. Simultaneously, convective downflows pull portions of the field lines downward. Turbulence twists and stretches the field lines. As the magnetic flux approaches the surface it spreads out. In the weaker $(10 \mathrm{kG})$ case, the downflows are able to confine the field to only a portion of the $48 \mathrm{Mm}$ wide domain, but in the stronger ( 20 and $40 \mathrm{kG}$ ) cases the field spreads over the entire domain at the surface. It is quickly swept by the diverging upflows to the boundaries of the granules and more slowly to the boundaries of mesogranule-scale structures (Figure 2). The emerging magnetic field distorts the granules, elongating them in the direction of the horizontal field. As the large loop in the $20 \mathrm{kG}$ case reaches the surface, it produces a large bipolar region (Figure 3) with a typical field strength of $2 \mathrm{kG}$ at continuum optical depth unity. The simulations have not yet run long enough to see if the supergranule-scale diverging flows will produce a magnetic network.
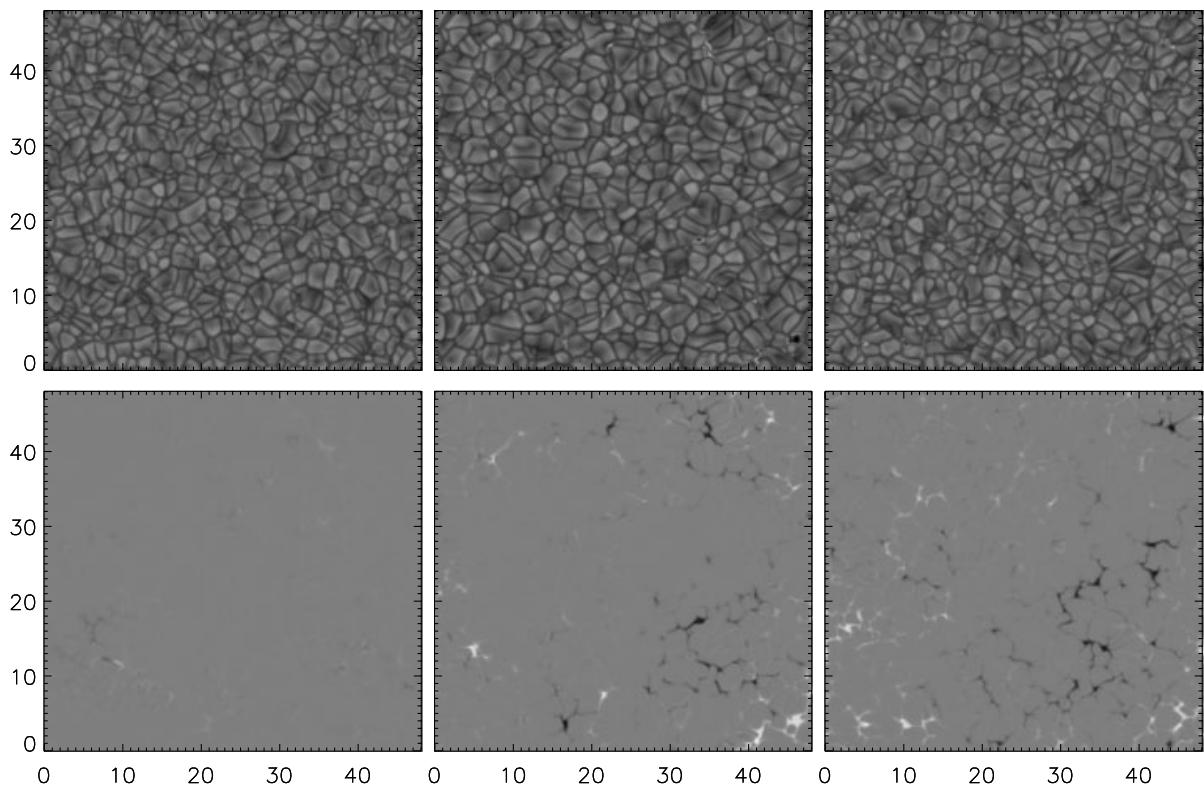

Figure 2 Vertical field (bottom row) and emergent continuum intensity (top row) at times 33, 40, and 48 hours after the $10 \mathrm{kG}$ field started to be introduced at the bottom. The range in the field strength is $[-2.8,2.84] \mathrm{kG}$. The range in the emergent continuum intensity is 0.5 to 2.0 times the average. 

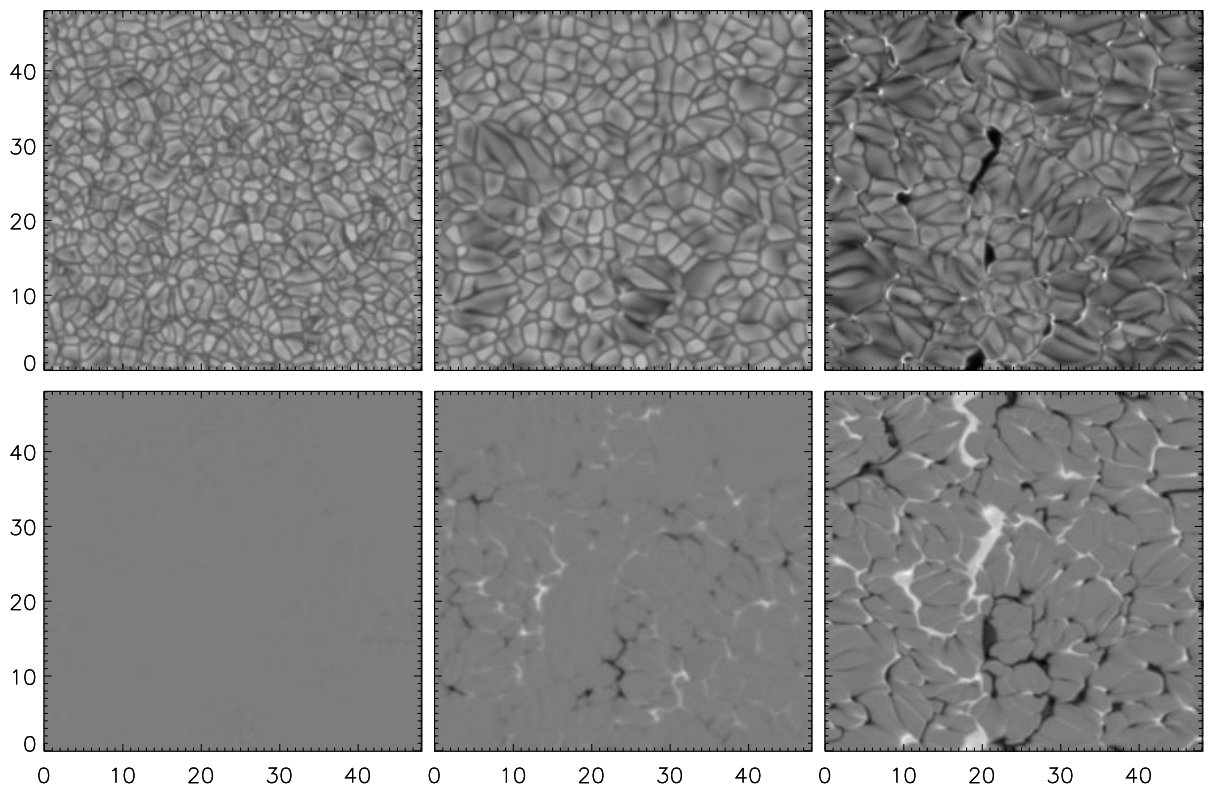

Figure 3 Vertical field (bottom row) and emergent continuum intensity (top row) at times 18, 20, and 23 hours after the $20 \mathrm{kG}$ field started to be introduced at the bottom. The range in the field strength is $[-3,3.4] \mathrm{kG}$. The range in the emergent continuum intensity is 0.25 to 1.9 times the average.
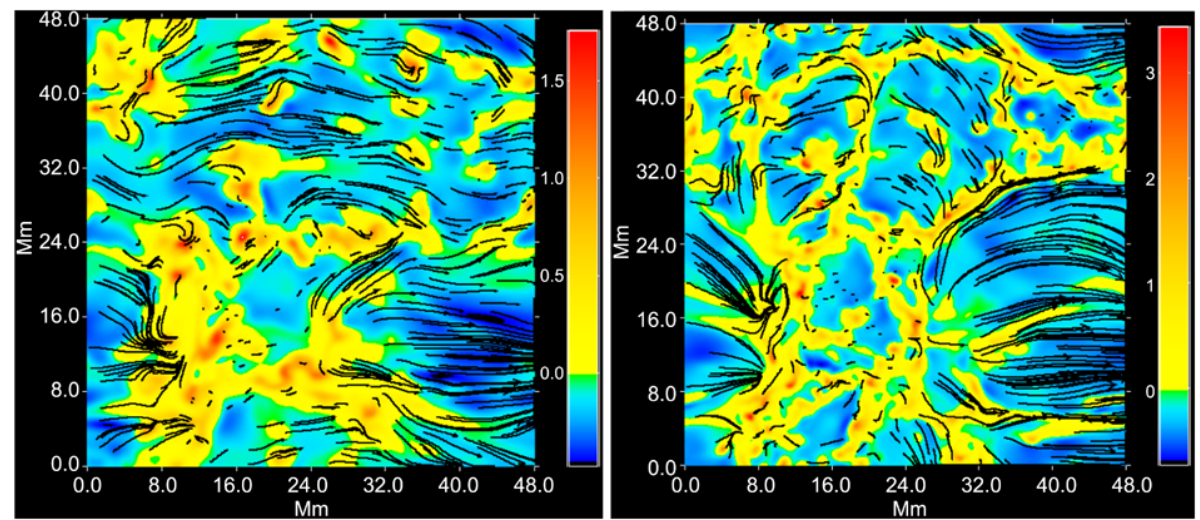

Figure 4 Vertical velocity (red and yellow downward, blue and green upward) and horizontal magnetic field during the initial rise through the non-magnetic atmosphere for the $20 \mathrm{kG}$ case at depth $8 \mathrm{Mm}$ and $4 \mathrm{Mm}$. At these intermediate depths the magnetic field is being advected in the upflows. The downflows are still confining the magnetic flux to deeper layers and so are mostly field-free at these depths. The field is slowly spreading horizontally with decreasing depth.

At the bottom of the domain, the horizontal magnetic field is entering the domain in the supergranule-scale upflows. These upflows, plus magnetic buoyancy, cause the field to rise to the surface. The downflows at these early times keep the field pinned down near the bottom of the domain. As a result, the downflows are nearly field-free and the magnetic field, even though untwisted, is confined to the upflows (Figure 4). As the surface is approached, the 

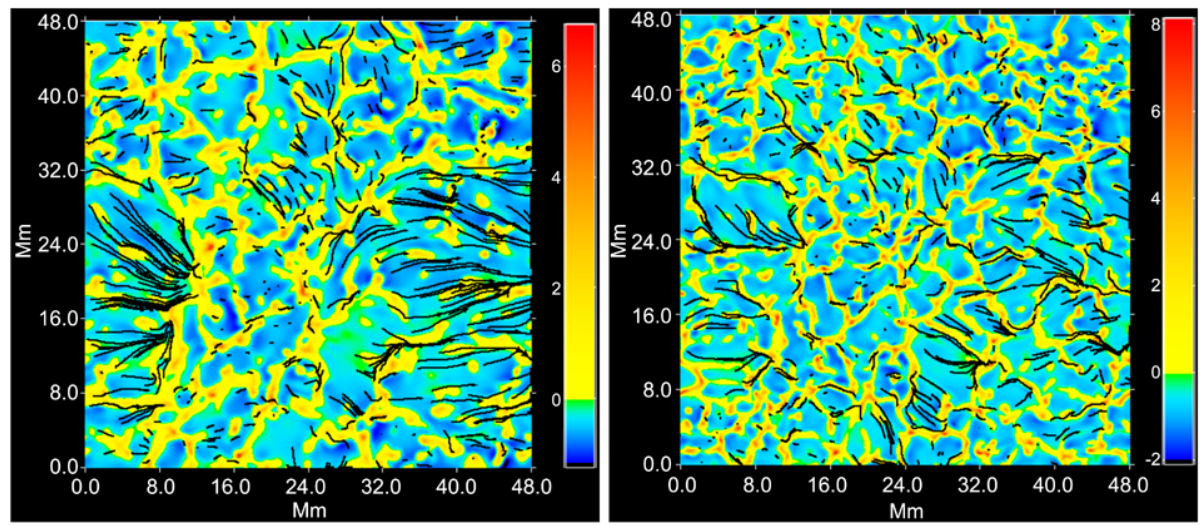

Figure 5 Vertical velocity (red and yellow downward, blue and green upward) and horizontal magnetic field during the initial rise through the non-magnetic atmosphere for the $20 \mathrm{kG}$ case at depth $2 \mathrm{Mm}$ and $1 \mathrm{Mm}$. Nearer the surface the magnetic field has spread farther and now almost surrounds the area of the large downflow at deeper levels.

Figure 6 Vertical velocity's horizontal pattern in the $48 \mathrm{Mm}$ wide simulation, at depths $2,4,8$, 12 and $16 \mathrm{Mm}$ below the surface. Blue and green are upflows. Red and yellow are downflows. The rms velocities are respectively $1.47,0.93,0.55,0.35,0.31$ and $0.24 \mathrm{~km} \mathrm{~s}^{-1}$. The size of the horizontal cellular patterns of the vertical velocity increases with increasing depth as the scale height increases.

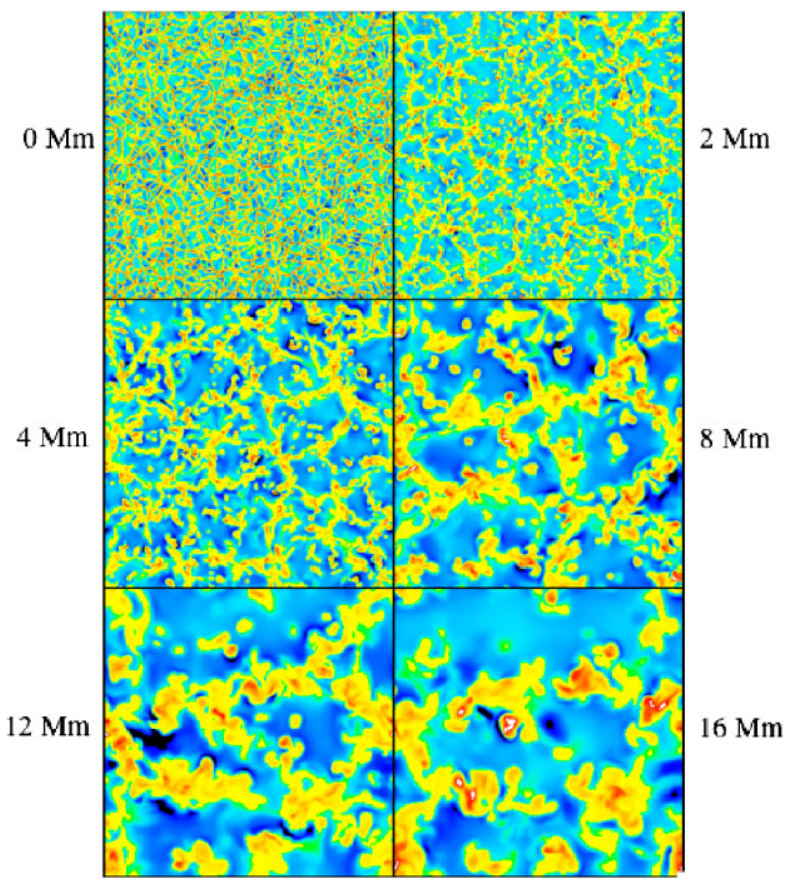

vertical velocity becomes broken up into smaller and smaller cells and the magnetic field is spread around. However, the area above the large, deep downflow still remains mostly field-free. It becomes ringed with field lines diverging from it on one side and converging toward it on the other (Figure 5). This is the manifestation of the very large loop structure that is produced (Figure 8). While the weaker field is rising, the large-scale downflow pattern at depth is changing and the magnetic field does not have such coherent large-scale loops. 


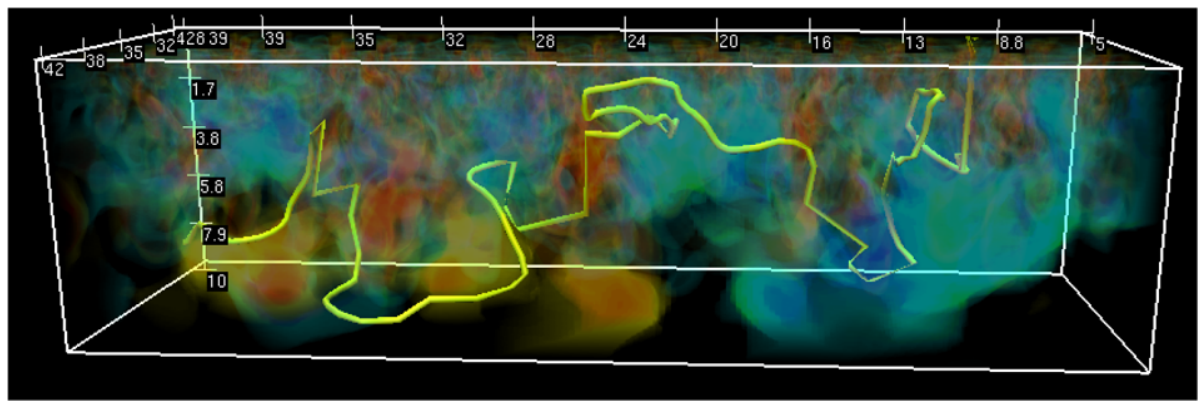

Figure 7 Example magnetic field line for the $10 \mathrm{kG}$ case, showing loop-like structure on many scales, from 2 to $20 \mathrm{Mm}$. Volume visualization is scaled vertical velocity (to make its visibility uniform with depth). Blue and green upwards, yellow and red downwards. Distance scales are in $\mathrm{Mm}$.

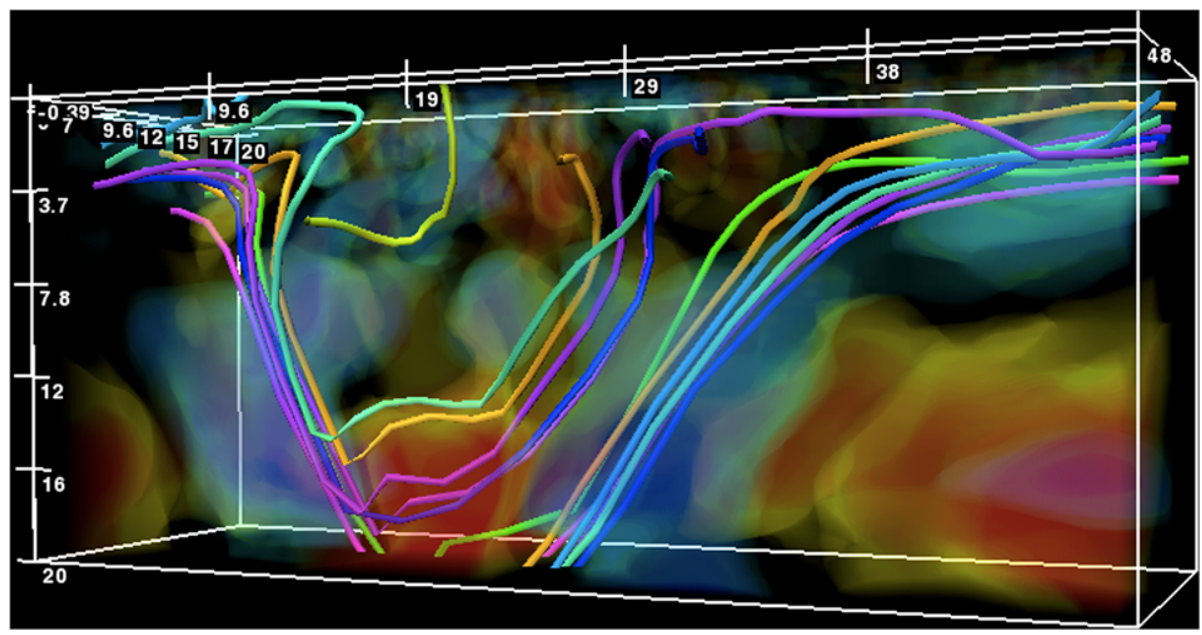

Figure 8 Large-scale magnetic loop extending the full domain in the $x$-direction (direction of the horizontal field entering the domain from below) and from the bottom at $20 \mathrm{Mm}$ to almost the surface. The volume rendering is the scaled vertical velocity (to make the flow at depth as visible as at the surface). Red and yellow are downflows and green and blue are upflows. The axis labels are megameters.

\section{Magnetic Topology}

The magnetic field is transported toward the surface in the upflows (assisted by magnetic buoyancy where the field strength is large enough) and pushed down in the downdrafts which creates $\Omega$ and U loops from the initially-horizontal field. Since the size of the horizontal cellular structure of convection flows decreases as the surface is approached (from supergranule size near the bottom to granule size near the surface, Figure 6) this produces loop structures over this range of scales as well (Figure 7).

An example of a large-scale loop structure spanning most of the $48 \mathrm{Mm}$ width and $20 \mathrm{Mm}$ depth is shown in Figure 8. The magnetic field was buoyant as well as being transported by the upflows and was pushed down in the downflows. An example of small loops near the surface is shown in Figure 9. The field lines close to the surface are very twisted on the scale of a few megameters. Note that the several field lines that loop over a granule interior connect very differently beneath the surface because of the turbulent convective motions. 


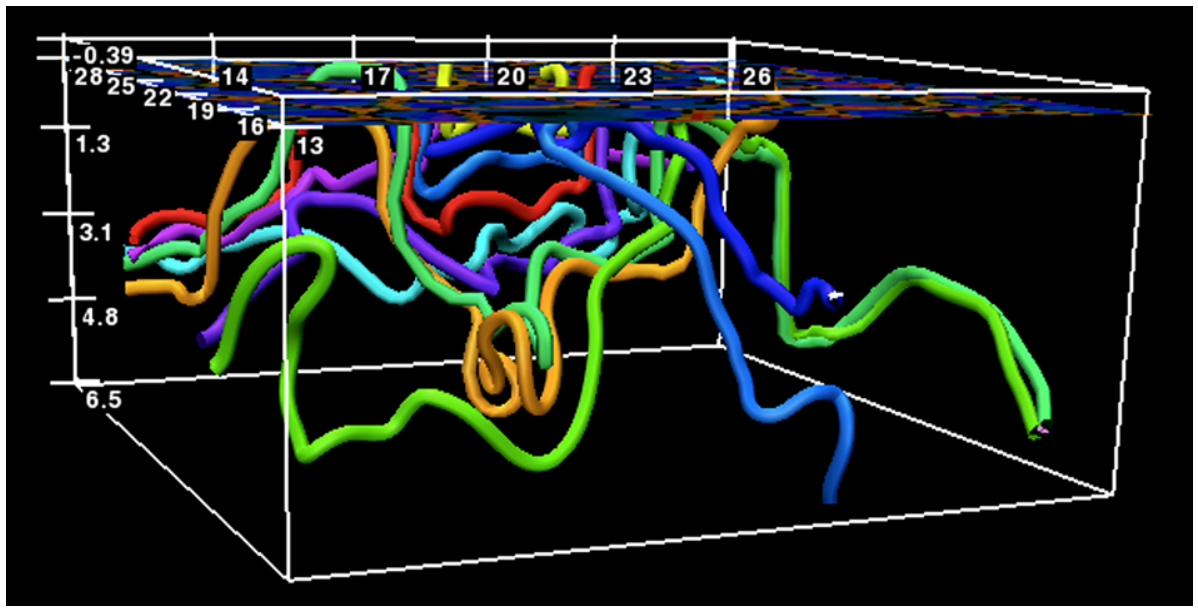

Figure 9 Small magnetic loops near the surface. The plane near the top is the visible surface with the vertical velocity pattern. Note that the field lines (yellow, green, red and blue) that are looping over a granule interior connect very differently beneath the surface. The vertical axis goes from $-0.39 \mathrm{Mn}$ at the top to $6.5 \mathrm{Mm}$ at the bottom of the figure.
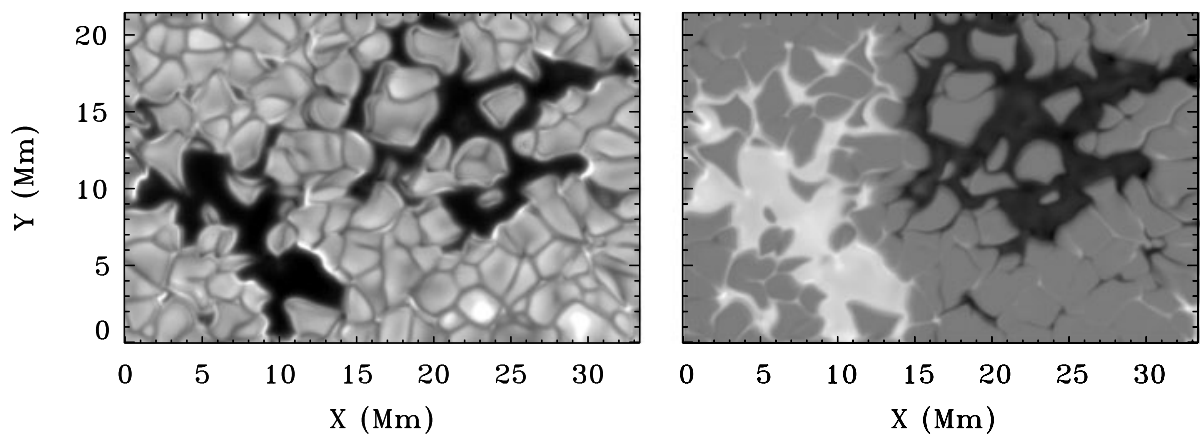

Figure 10 Emergent continuum intensity (left) and vertical magnetic field at continuum optical depth unity (right) in a plage region of the $20 \mathrm{kG}$ case.

In the $20 \mathrm{kG}$ case, there is enough magnetic flux that it becomes concentrated in large regions to field strengths of $2 \mathrm{kG}$ at continuum optical depth unity and produces pores (Figure 10). The intensity in the pores is $1 / 4$ of the average intensity.

The magnetic field lines in this pore region are generally nearly vertical at the surface and have a U-shape below the surface (Figure 11). The opposite-polarity field lines at the surface generally connect at large depths (greater than $10 \mathrm{Mm}$ ) below the surface.

In all three cases the magnetic-energy density is larger than the fluid kinetic-energy density below $10 \mathrm{Mm}$. However, this is not necessarily an argument against such strong fields, since they could be confined because they are in pressure equilibrium with their surroundings. These preliminary results, however, suggest that the magnetic field can not be as strong as $20 \mathrm{kG}$ at $20 \mathrm{Mm}$ unless it has a lower entropy then the field-free plasma, because magnetic bubbles develop, due to the large magnetic buoyancy, and produce large, hot, bright granules at the surface, which are not seen. More cases need to be calculated and compared with observations to clarify the properties of the magnetic field at $20 \mathrm{Mm}$ depth. 

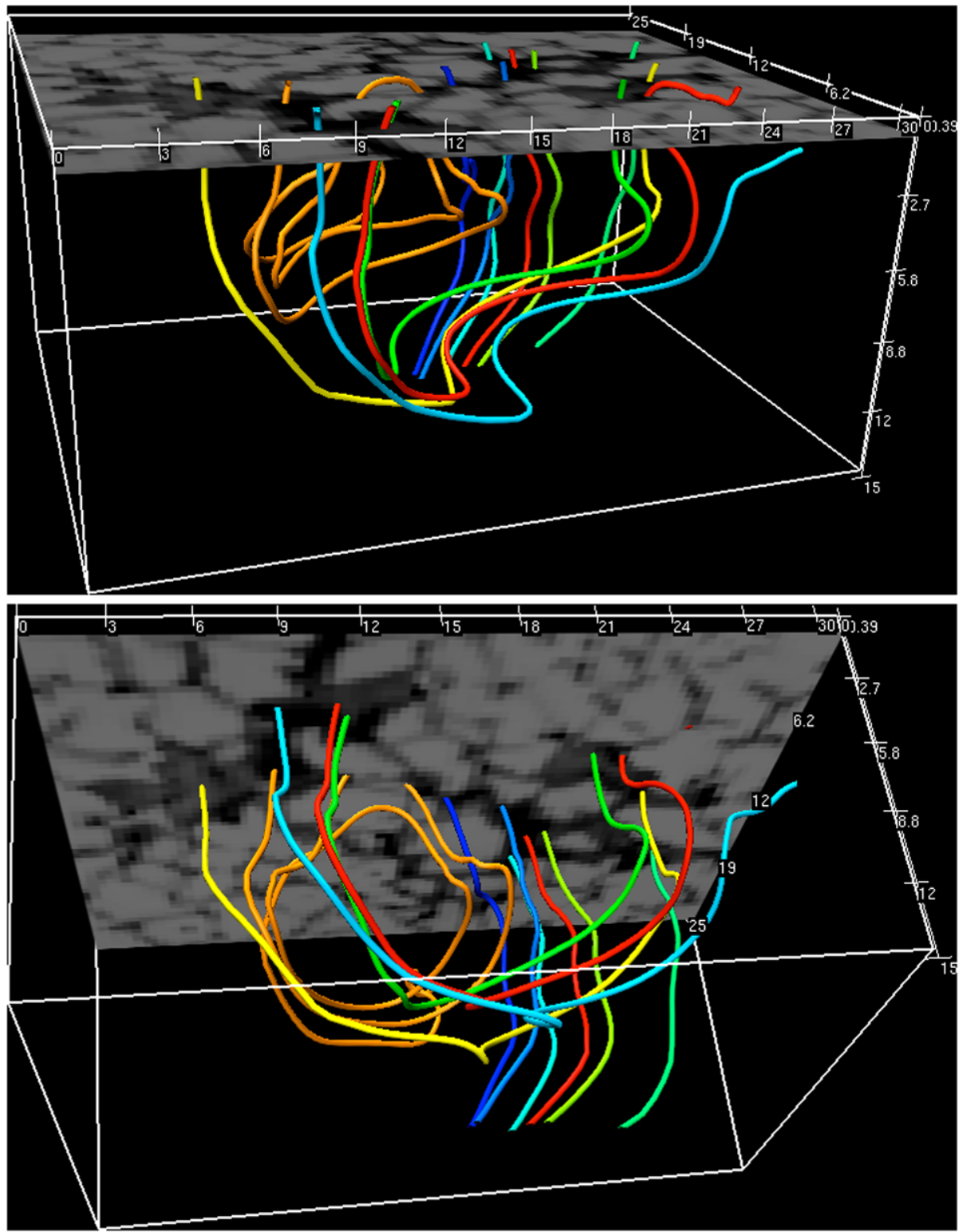

Figure 11 Magnetic field lines and emergent intensity in bipolar pore region looked at from above and below. In this case many field lines are connected in a U-configuration. Axes scales are in Mm.

\section{Application to Helioseismology}

$f$ - and $p$-mode waves are excited in the simulation and can be used for testing and refining local-helioseismic techniques. Figure 12 shows an example of waves generated near the surface, propagating into the interior and refracted by the increasing sound speed with depth. We have made preliminary $k-\omega$ diagrams and compared the magnetic and nonmagnetic 


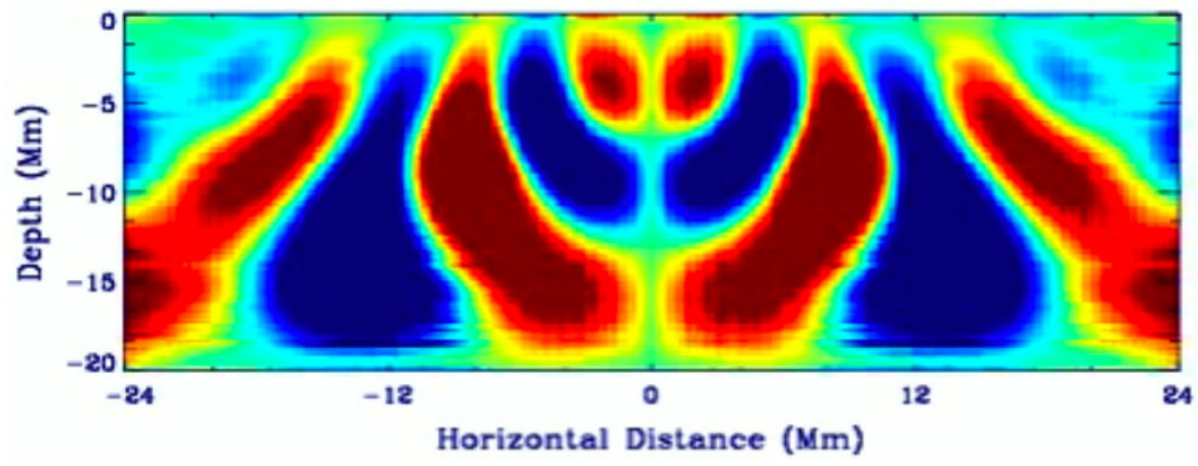

Figure 12 Acoustic waves (visualized by the density fluctuation that they produce) in the non-magnetic simulation propagating into the interior and refracted by the increasing sound speed with depth as calculated by J. Zhao, following the procedure described in Zhao et al. (2007).
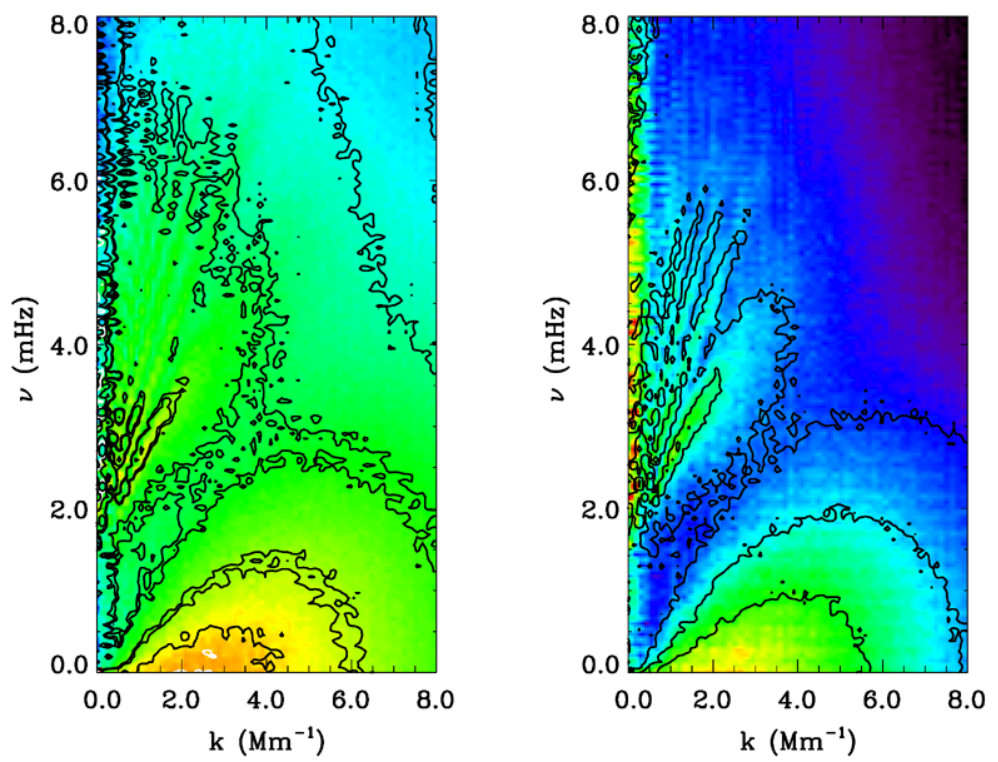

Figure $13 k-\omega$ diagram comparing non-magnetic and magnetic $(20 \mathrm{kG})$ case. On the left the magnetic-case contours are overlaid on the non-magnetic image and vice versa on the right. There is no shift in the mode ridges and the image on the left looks like the contours on the right and vice versa, so that there do not appear to be any differences produced so far in this calculation.

cases. For the early stage of the flux emergence studied so far, there are no discernable differences (Figure 13).

\section{Discussion}

The results we have reported here concern only the initial rise of uniform, untwisted, horizontal magnetic flux through the near-surface, nonmagnetic, solar convection zone. The 
main result, so far, is that fields as strong as $20 \mathrm{kG}$ at $20 \mathrm{Mm}$ depth are not possible unless they have lower entropy than the surrounding field-free plasma. Using these simulations, we will continue to investigate how the magnetic flux evolves over time, how the pores evolve, whether sunspots form, whether a magnetic network is produced and what its properties are, how flux emerges through an existing magneto-convecting surface layer, how these simulations compare with observations, and what constraints can be placed on the properties of the magnetic field near depths of $20 \mathrm{Mm}$. The $f$ - and $p$-modes excited in these simulations will be used to investigate local-helioseismic inversion procedures.

Acknowledgements The calculations reported here were performed with resources provided by the NASA High-End Computing (HEC) Program through the NASA Advanced Supercomputing (NAS) Division at Ames Research Center. They would not have been possible without this generous support. Graphics were produced with VAPOR \{www.vapor.ucar.edu\} and IDL \{www.ittvis.com/idl\}. This work was supported by NASA grants NNX07AO71G, NNX07AH79G and NNX08AH44G, and NSF grant AST0605738, which is greatly appreciated.

Open Access This article is distributed under the terms of the Creative Commons Attribution Noncommercial License which permits any noncommercial use, distribution, and reproduction in any medium, provided the original author(s) and source are credited.

\section{References}

Abbett, W.P., Fisher, G.H., Fan, Y., Bercik, D.J.: 2004, The dynamic evolution of twisted magnetic flux tubes in a three-dimensional convecting flow. II. Turbulent pumping and the cohesion of $\Omega$-loops. Astrophys. J. 612, $557-575$. doi: $10.1086 / 422444$.

Asplund, M., Nordlund, Å., Trampedach, R., Allende Prieto, C., Stein, R.F.: 2000, Line formation in solar granulation. I. Fe line shapes, shifts and asymmetries. Astron. Astrophys. 359, 729-742.

Caligari, P., Moreno-Insertis, F., Schüssler, M.: 1995, Emerging flux tubes in the solar convection zone. 1: Asymmetry, tilt, and emergence latitude. Astrophys. J. 441, 886-902. doi:10.1086/175410.

Cheung, M.C.M., Moreno-Insertis, F., Schüssler, M.: 2006, Moving magnetic tubes: fragmentation, vortex streets and the limit of the approximation of thin flux tubes. Astron. Astrophys. 451, 303-317. doi:10.1051/0004-6361:20054499.

Cheung, M.C.M., Schüssler, M., Moreno-Insertis, F.: 2007, Magnetic flux emergence in granular convection: radiative MHD simulations and observational signatures. Astron. Astrophys. 467, 703-719. doi:10.1051/0004-6361:20077048.

Cheung, M.C.M., Schüssler, M., Tarbell, T.D., Title, A.M.: 2008, Solar surface emerging flux regions: A comparative study of radiative MHD modeling and Hinode SOT observations. Astrophys. J. 687, $1373-$ 1387. doi:10.1086/591245.

Choudhuri, A.R., Gilman, P.A.: 1987, The influence of the Coriolis force on flux tubes rising through the solar convection zone. Astrophys. J. 316, 788 - 800. doi:10.1086/165243.

Dorch, S.B.F.: 2003, Buoyant magnetic flux ropes and convection: Evolution prior to emergence. In: Brown, A., Harper, G.M., Ayres, T.R. (eds.) The Future of Cool-Star Astrophysics: 12th Cambridge Workshop on Cool Stars, Stellar Systems, and the Sun 12, University of Colorado, Boulder, 186-195.

Fan, Y.: 2009, The emergence of a twisted flux tube into the solar atmosphere: Sunspot rotations and the formation of a coronal flux rope. Astrophys. J. 697, 1529-1542. doi:10.1088/0004-637X/697/2/1529.

Fan, Y., Fisher, G.H., McClymont, A.N.: 1994, Dynamics of emerging active region flux loops. Astrophys. J. 436, 907 -928. doi:10.1086/174967.

Feautrier, P.: 1964, A procedure for computing the mean intensity and the flux. SAO Special Report, Smithsonian Astrophys. Obs, Cambridge 167, 80.

Kennedy, C.A., Carpenter, M.H., Lewis, R.M.: 1999, Low storage, explicit Runge - Kutta schemes for compressible Navier-Stokes equations. ICASE Report, NASA 99-22, 1.

Martínez-Sykora, J., Hansteen, V., Carlsson, M.: 2008, Twisted flux tube emergence from the convection zone to the corona. Astrophys. J. 679, $871-888$. doi:10.1086/587028.

Martínez-Sykora, J., Hansteen, V., Carlsson, M.: 2009, Twisted flux tube emergence from the convection zone to the corona. II. Later states. Astrophys. J. 702, 129 - 140. doi:10.1088/0004-637X/702/1/129.

Moreno-Insertis, F.: 1986, Nonlinear time-evolution of kink-unstable magnetic flux tubes in the convective zone of the sun. Astron. Astrophys. 166, $291-305$. 
Moreno-Insertis, F., Caligari, P., Schüssler, M.: 1994, Active region asymmetry as a result of the rise of magnetic flux tubes. Solar Phys. 153, 449-452. doi:10.1007/BF00712518.

Nordlund, Å.: 1982, Numerical simulations of the solar granulation. I-Basic equations and methods. Astron. Astrophys. 107, $1-10$.

Stein, R.F., Nordlund, Å.: 1998, Simulations of solar granulation. I. General properties. Astrophys. J. 499, 914. doi:10.1086/305678.

Stein, R.F., Nordlund, Å.: 2003, Radiative transfer in 3d numerical simulations. In: Hubeny, I., Mihalas, D., Werner, K. (eds.) Stellar Atmosphere Modeling CS-288, Astron. Soc. Pac., San Francisco, 519.

Vögler, A., Bruls, J.H.M.J., Schüssler, M.: 2004, Approximations for non-grey radiative transfer in numerical simulations of the solar photosphere. Astron. Astrophys. 421, 741-754. doi:10.1051/0004-6361:20047043.

Zhao, J., Georgobiani, D., Kosovichev, A.G., Benson, D., Stein, R.F., Nordlund, Å.: 2007, Validation of time-distance helioseismology by use of realistic simulations of solar convection. Astrophys. J. 659, 848 - 857. doi:10.1086/512009. 